\title{
Neutrino Flux Analysis and Monitoring for Power Improvements in NuMI
}

Nilay Bostan ${ }^{a}$, K. Yonehara, L. A. Soplin, A. Wickremasinghe ${ }^{b}$, P. Snopok, Y. Yu ${ }^{c}$, A. Bashyal ${ }^{d}$

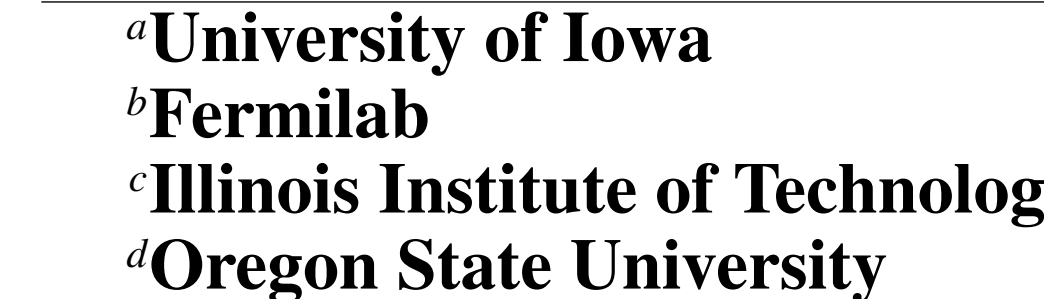

Introduction

The determination of the neutrino flux from accelerator neutrino beams exhibits ments. These experiments provide the measurements of the neutrino oscillatio parameters, the mass hierarchy, and the CP phase with high sensitivity. The cur-
rent flux predictions for the on-axis and off-axis NuMI (Neutrinos at the Main Injector) neutrino detector locations depend on GEANT4 based beam simulatior code called G4NuMI. The current simulation uses the new NuMI target, which has $1.5 \mathrm{~mm}$ spot size and it is expected to get $900 \mathrm{~kW}$ and even more in the up
coming years. In this work, for this new target system, we study the neutrin flux corresponding to the muon energy thresholds seen by the Muon Monito for FTFP BERT hadronic model and investigate he neytrino flux predictior at the on-axis and off-axis NuMl netu using G4NuMI beam simulation. We al and QGSP BERT hadronic models by 1 ackage to Predict the Flux) to the neutrin
present the application of the PPFX (Package flux at the on-axis and off-axis NuMI detector locations for FTFP BERT hadron model. Finally, we investigate the neutrino spectrum at the NuMI neutrino dete tor locations that come from $\pi^{+}$through the focusing $\mathrm{c}$
based on G4NuMI with 50M protons on target (POT).

A brief description of the NuMI beamline

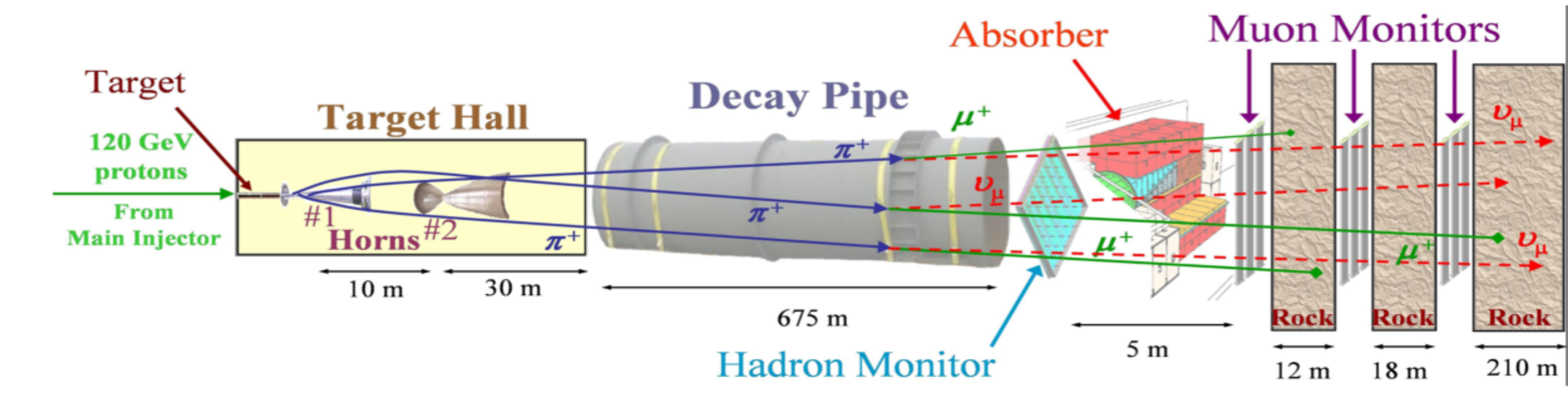

Figure 1: The diagram of the NuMI beamline [1]

- $120 \mathrm{GeV} / \mathrm{c}$ momentum protons are delivered by the Fermilab Main Injector dominantly pions. The pions are focused by a pair of pulsed horn magnets into the decay pipe. The pions then decay to muons and muon neutrinos.

- The Hadron Monitor measures the spatial distribution of the uninteracted protons and undecayed pions, after which they are stopped in the Hadron $\mathbf{A b}$ -

- The muons penetrate through the absorber and some rock. Their spatial distri-

to detectors.

The comparison between new and old target system

New vs Old Target

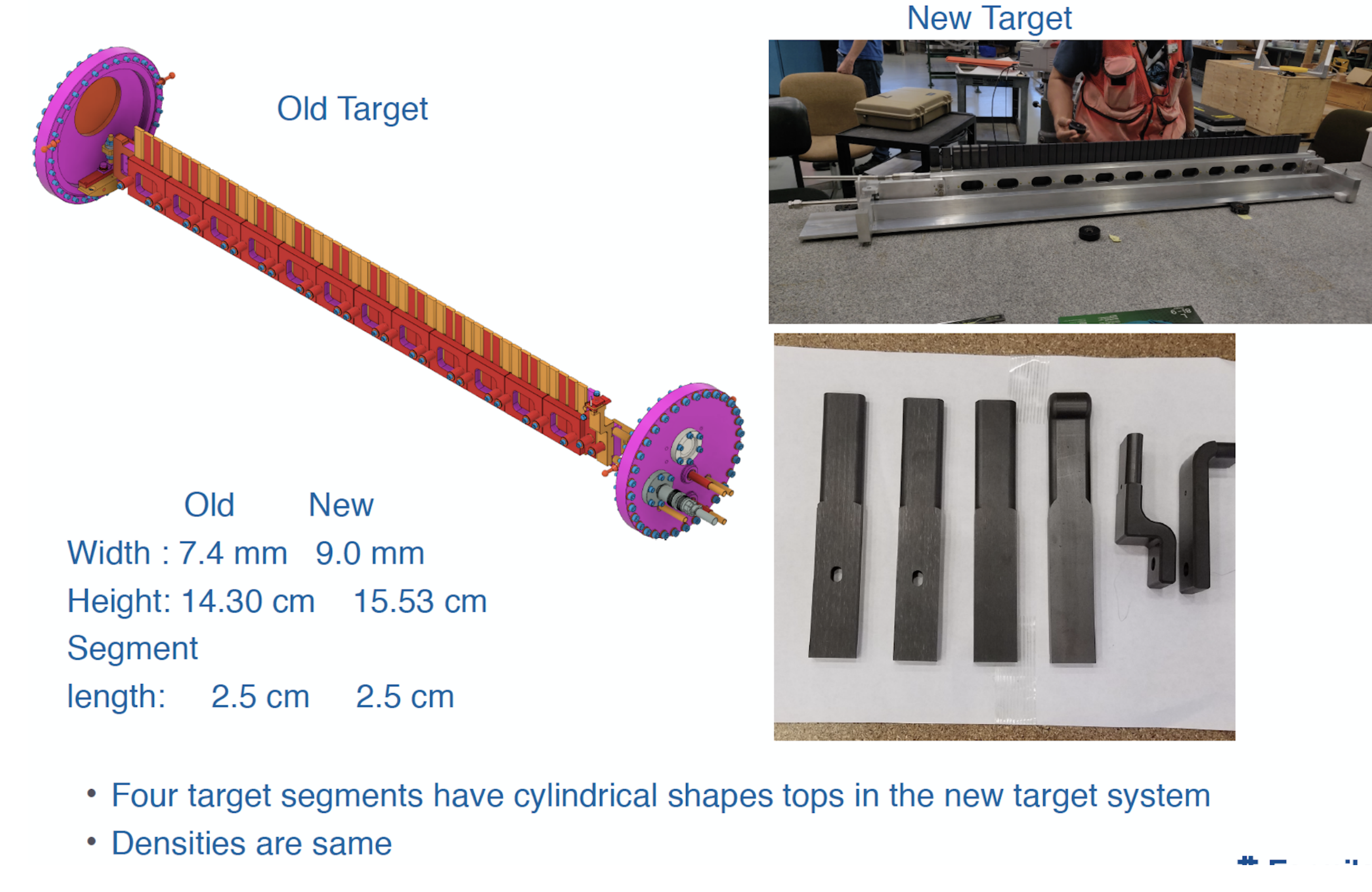

New target: $1.5 \mathrm{~mm}$ spot size and it is expected to get $900 \mathrm{~kW}$ and even more

.Old

Neutrino energy and flux

The energy and flux of the neutrinos depend on the decay angle $\left(\theta_{\nu}\right)$. Assuming that the neutrinos are moving in a near forward direction, for a two-body decay,
the neutrino energy can be calculated. The neutrino energy and flux are defined as

$$
E_{\nu} \approx \frac{\left(1-\frac{m_{\mu}^{2}}{M^{2}}\right) E_{\pi(K)}}{1+\gamma^{2} \tan ^{2} \theta_{\nu}}, \quad \Phi_{z}=\left(\frac{2 \gamma}{1+\gamma^{2} \theta_{\nu}^{2}}\right)^{2} \frac{A}{4 \pi z^{2}}
$$

respectively. Here, muon and meson masses are represented by $m_{\mu}$ and $\mathbf{M}$, resper factor, where $\gamma=\underline{E}_{\pi(K)}, \theta_{\nu}$, decay angle between the pions (kaons) and the prr duced neutrino directions, $A$, transverse area of the detector and $z$, the distan between the decay point and the detector location
Neutrino flux according to the thresholds of muon energy seen by the Muon Monitors

We show that the neutrino flux for on-axis and off-axis NuMI neutrino detector

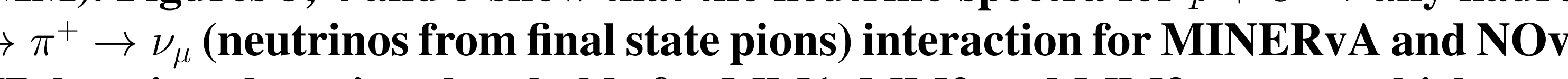

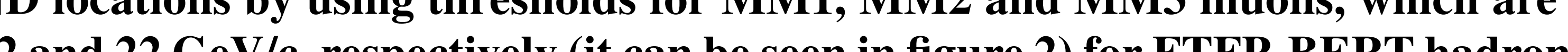
model.
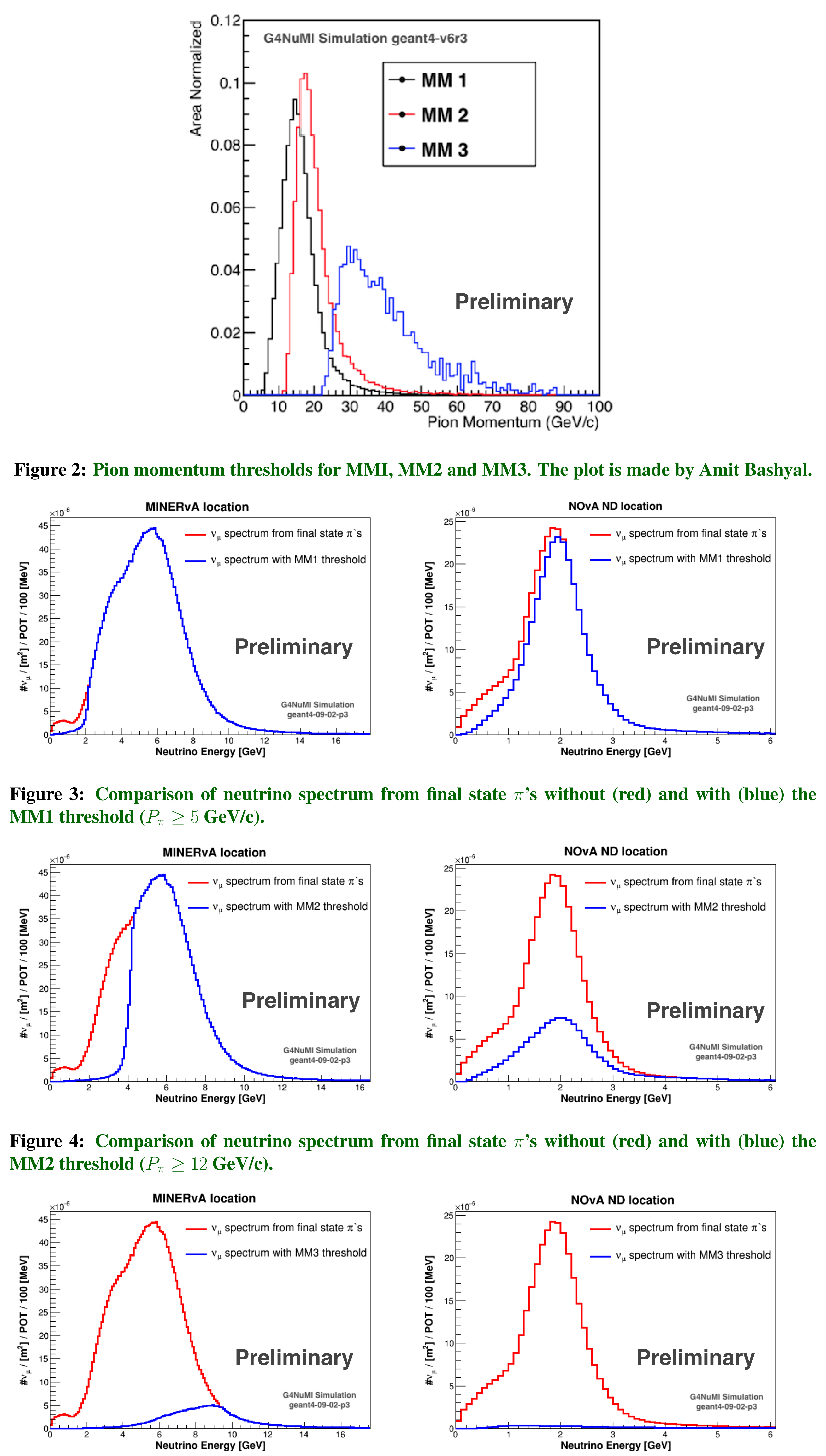

\section{rigure 5: Comparison of neev
MMM threshold $\left(P_{\pi} \geq 22 \mathrm{GeV} / \mathrm{c}\right)$}

Three Each muon monitor contains $9 \times 9$ arrays of ionization chambers. Each ioniz chambers are filled with He gas. Furthermore, muon monitors might $3 \mathrm{~mm}$. The cerse are correlated $\left(\pi^{+} \rightarrow \mu^{+}+\nu_{\mu}\right)$. The plots in Figs. 3, $\mathbf{4}, \mathbf{5}$ show the correlation Neutrino spectra at the on-axis and off-axis NuMI neutrino detector locations

Neutrino spectra for $p+C \rightarrow \pi^{+} \rightarrow$ any interaction or decay $\rightarrow \nu_{\mu}$ (secondary pi-
ons to final state neutrinos) for FTFP BERT and QGSP BERT hadronic models are shown here. Figure 6 shows the results at the MINERva detector location, ffe or MINERvA and $2 \mathrm{GeV}$ for NOvA ND locations and the ratio between FTH and QGSP is $\approx 0.9$ for MINERvA detector and $\approx 0.85$ for NOvA ND location around focusing peak.

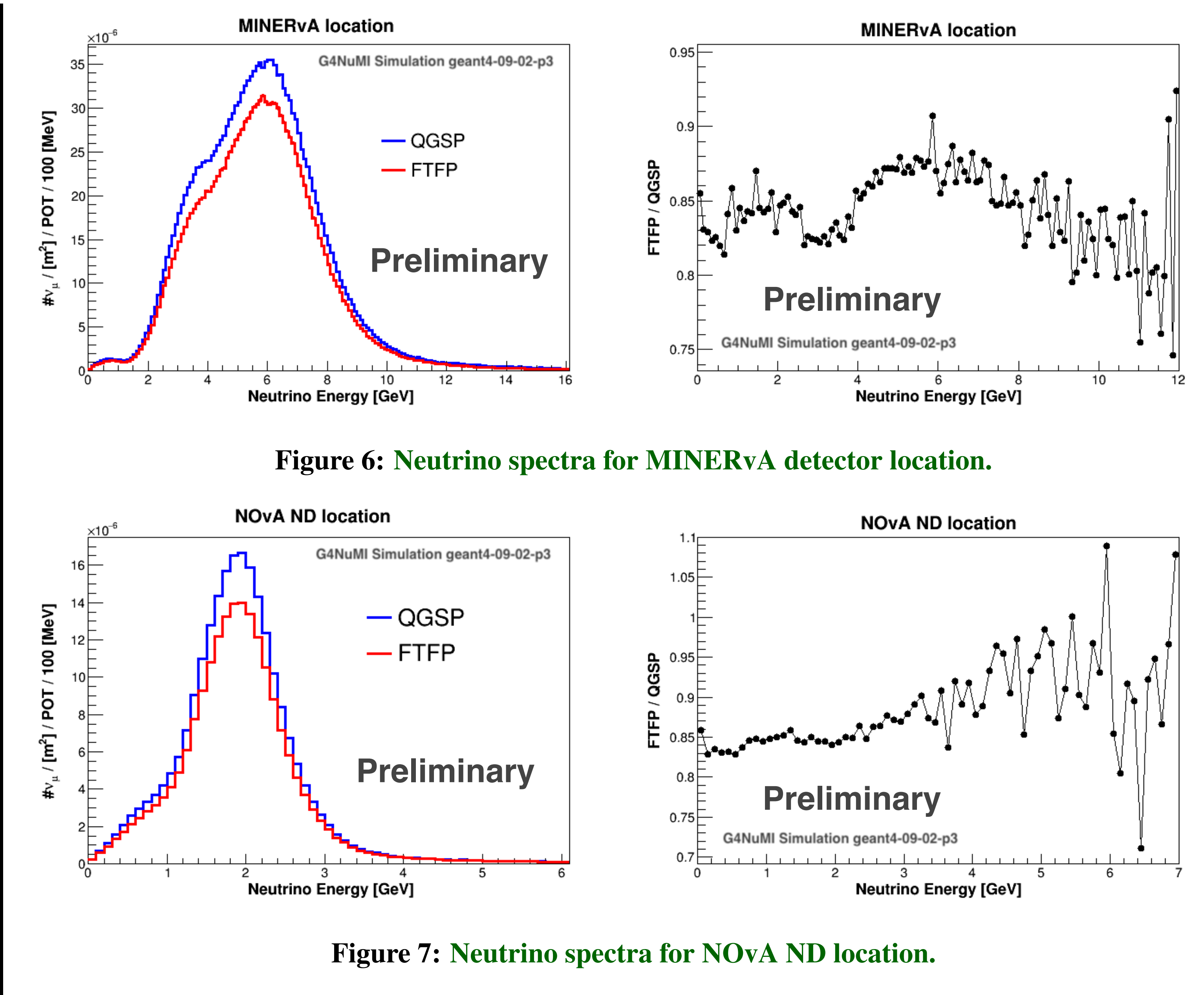

Flux correction by thin target datasets

We compare the impact of hadron production model to external data (NA49) by existing thin target datasets for FTFP BERT hadronic model with the 'multiuniverse' technique applied to a neutrino at generation event by event [2]. Figure 8 shows the ratio of PPFX to no PPFX for $\nu_{\mu}$ spectra at MINERva detector and tion, average flux, central value) in these plots.
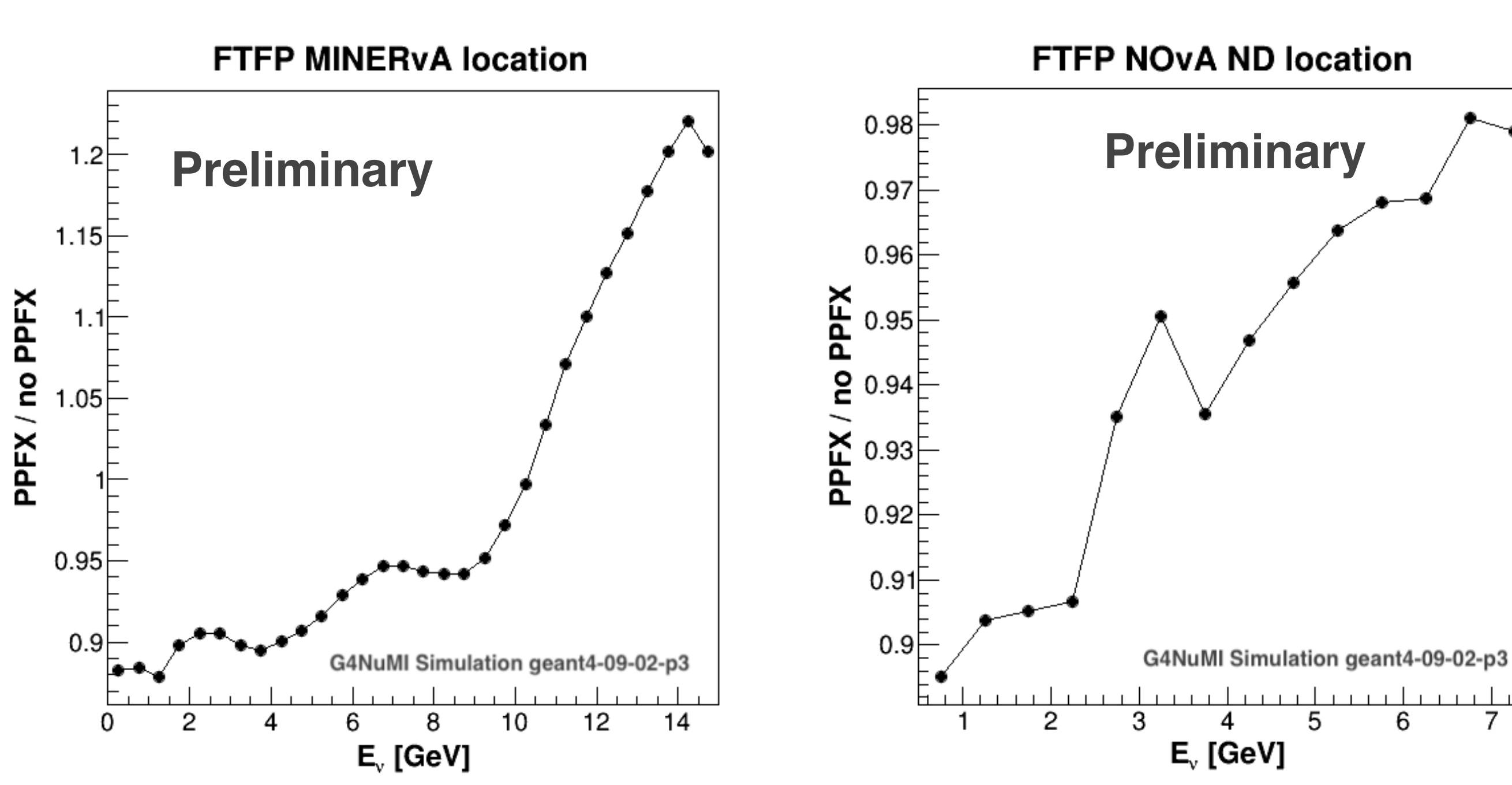

Figure
tions.
According

According to feres 8 ,

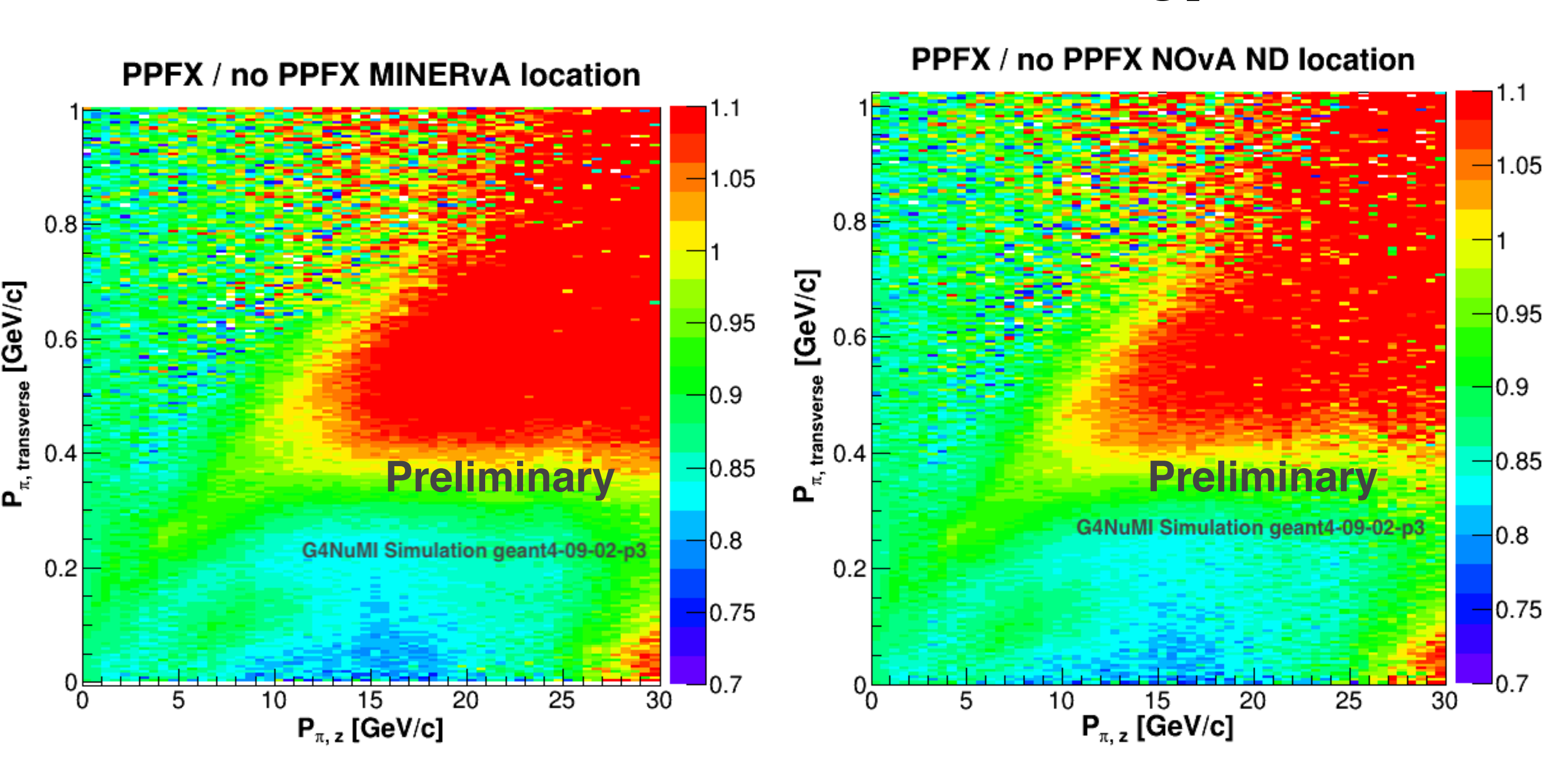

Figure 9: The ratio of $P$ P
momenta distributions.

Figure 9 shows the distribution of the wide range of $P_{z}$ vs. $P_{T}$ of secondary pions. PPFX to no PPFX is $\approx 0.9$. Given the upcoming new data, people would like to extract cross sections for both QGSP and FTFP model and targets and projectiles results that are given by figures 8 and 9 can help establish relations vith the works and our future studies about PPFX.

Focusing components for NuMI new target at NOvA ND and MINERvA locations

We present the focusing components for NuMI, by taking into account the NuMI new target system and FTFP hadronic model at NOvA ND and MINERvA loca-
tions. To understand the effect of the focusing system, we split the neutrino flux

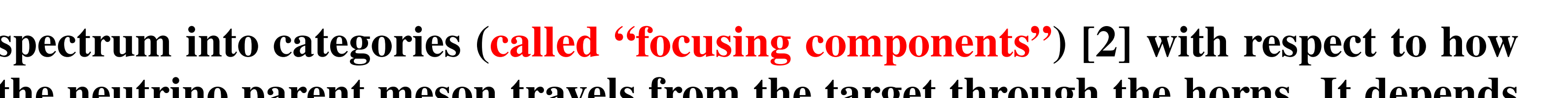
ine neutrino pact meson travels the the mesons with respect to their longitudinal momentum (figure 10).
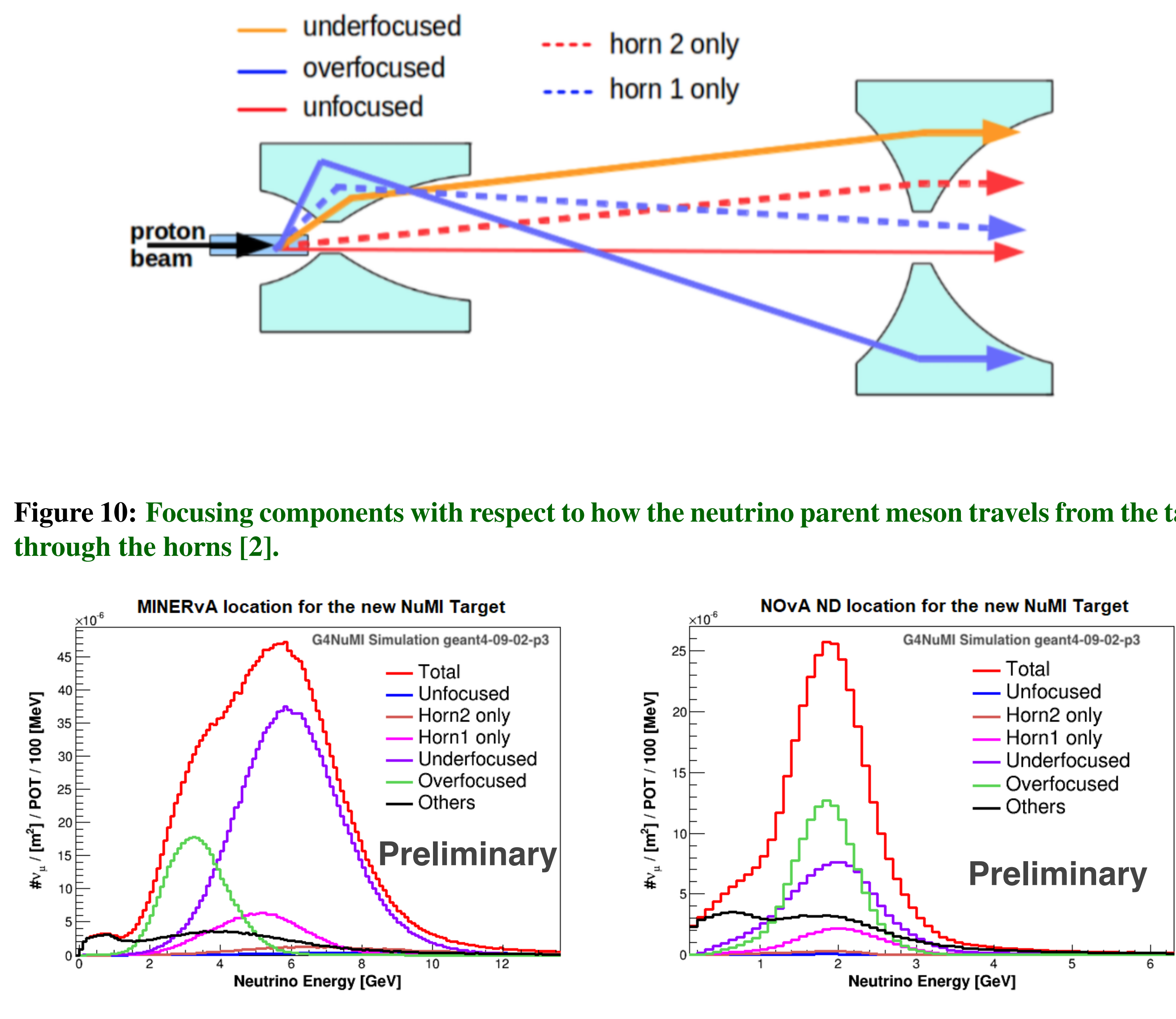

ligure 1 1.
parent.
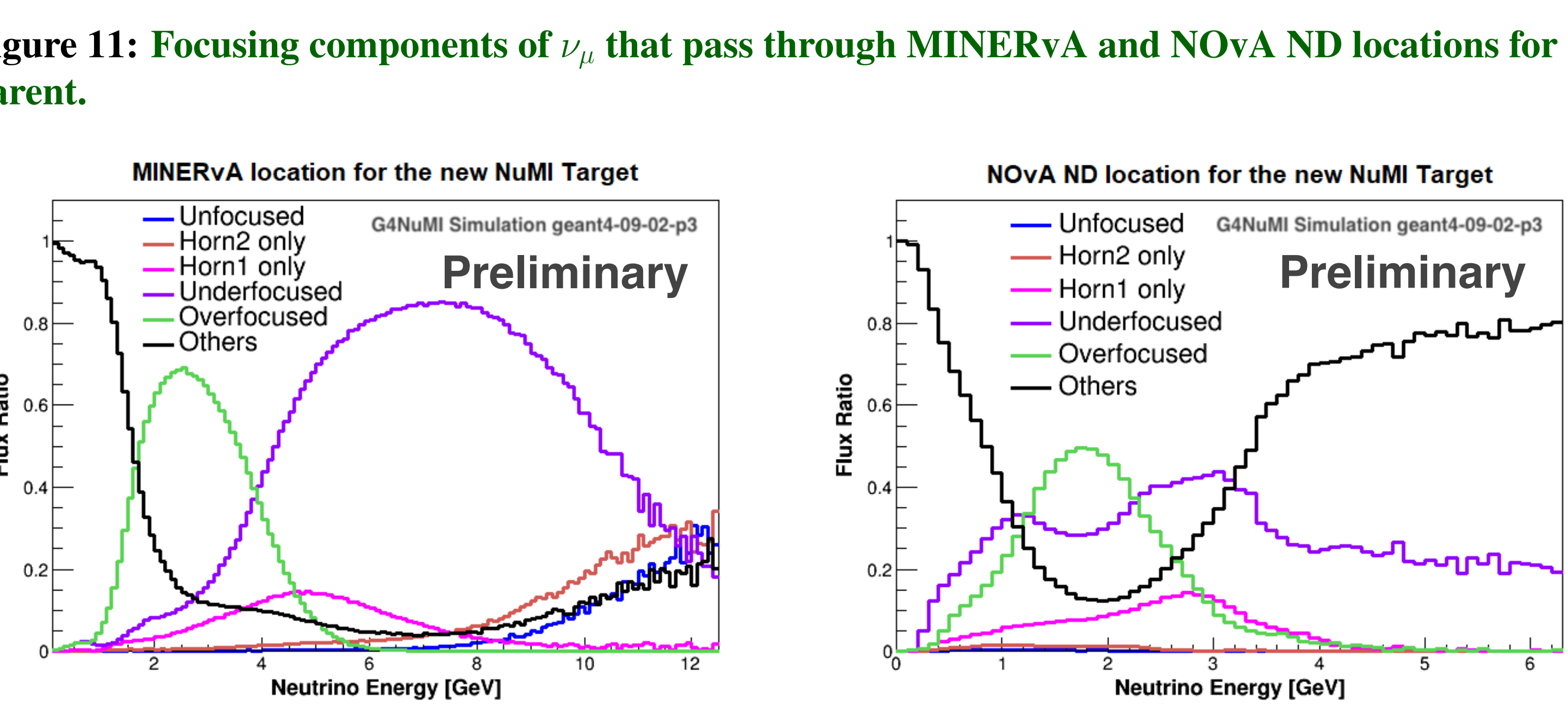

\section{Figure 12: Beam focus
tions for $\pi^{+} \nu_{n}$ parent.} extend the neutrino energy to $20 \mathrm{GeV}$ for $\pi^{+}$, as we go from higher to lower mo-
ent mentum, unfocused, underfocused and overfocused pion decays populate the $\nu_{\mu}$ spectra narrow momentum range values allow the pions to be focused by just one
horn. The very low energy neutrinos come mostly from pions born outside of the arget ('others') from secondary and tertiary hadrons.

\section{Conclusions}

- The NuMI beamline and the new NuMI target are described

- Neutrino flux dependence on the hadronic models at the on-axis and off-axi

Pion momentum thresholds for MM1, MM2, and MM3 are established at 5, 12

locations

- Focusing effects were studied for $\pi^{+} \nu_{\mu}$ parent using FTFP hadronic model

\section{REFERENCES}

[1] Zwaska, Robert Miles. Accelerator systems and in
NuMI neutrino beam. United States. doi: 10.2172/879065.

[2] Aliaga Soplin, Leonidas. Neutrino Flux Prediction for the NuMI Beamline

Acknowledgements

NB wish to thank R. Zwaska, J. M. Nachtman and Y. Onel for their contribuBostan is also supported by fellowship by Republic of Turkey Ministry of National Education. 